\title{
Proyecto de Factibilidad Negocio de Bar - Safari Licores en Azogues
}

\section{Feasibility Project Bar Business - Safari Liquors in Azogues}

\author{
Alberto Esteban Mogrovejo Lazo*¹ ${ }^{1}$ Javier Alfonso Villavicencio Machado ${ }^{1}$; Cristóbal Oswaldo Aguilar Aguilar ${ }^{1}$ \\ ${ }^{1}$ Investigador independiente, Ecuador \\ *beteml@hotmail.com
}

DOI: https://doi.org/10.26871/killkanasocial.v4i3.657

\begin{abstract}
Resumen
Safari Licores es una empresa cuencana que se dedica al expendio de bebidas alcohólicas en instalaciones que tienen adecuaciones de un bar para la comodidad de sus clientes, esta 15 años dentro de este mercado en la ciudad de Cuenca lo que demuestra su experiencia sobre el negocio; analizando la posibilidad de extenderse con una sucursal de las mismas características en la ciudad de Azogues aprovechando la igualdad de las características culturales y de idiosincrasia de las dos ciudades; la problemática que se ha encontrado para desarrollar esta investigación es que Azogues no tiene un bar con las siguientes características; que sea un punto de encuentro entre amigos o conocidos donde no solamente se tome alcohol sino se pueda disfrutar de música en vivo, de la transmisión de eventos deportivos nacionales e internacionales; el objetivo es inaugurar un comercio de estas características en una ciudad con un crecimiento demográfico importante y una alza en el comercio en actividades económicas como restaurants, bares, licoreras especialmente en la zona sur de la ciudad; la investigación es de tipo mixta; utiliza datos cualitativos para conocer los gustos y recomendaciones sobre este mercado de la población azogueña y cuantitativos para establecer la demanda de este tipo de negocios y determinar su operatividad; con los resultados obtenidos en el Valor Actual Neto (VAN) US\$ 7,519.72 Tasa Interna de Retorno (TIR) 28.69\%; que está por encima de la tasa media del mercado, su beneficio costo $(\mathrm{B} / \mathrm{C})$ en 1.37 por encima de 1; por lo cual se recomienda la viabilidad del proyecto.
\end{abstract}

Palabras clave: comercio, demanda, emprendimiento, satisfacción.

\begin{abstract}
Safari Liquors is a Cuenca company that is dedicated to the sale of alcoholic beverages in facilities that have adaptations of a bar for the convenience of its customers, this 15 years within this market in the city of Cuenca which demonstrates its experience on the business; analyzing the possibility of extending with a branch of the same characteristics in the city of Azogues taking advantage of the equality of cultural and idiosyncrasy characteristics of the two cities; The problem that has been found to develop this research is that Azogues does not have a bar with the following characteristics; It is a meeting point between friends or acquaintances where not only alcohol is drunk but you can enjoy live music, the transmission of national and international sporting events; The objective is to inaugurate a trade of these characteristics in a city with significant population growth and an increase in trade in economic activities such as restaurants, bars, liquor stores, especially in the southern part of the city; the research is mixed type; it uses qualitative data to know the tastes and recommendations on this market of the population of Azerbaijan and quantitative to establish the demand for this type of business and determine its operation; with the results obtained in the Net Present Value (NPV) US \$ 7,519.72 Internal Rate of Return (IRR) 28.69\%; which is above the average market rate, its cost benefit $(B / C)$ at 1.37 above 1; Therefore, the viability of the project is recommended.
\end{abstract}

Keywords: trade, entrepreneurship, satisfaction, demand.

\section{Introducción}

En la ciudad de Azogues; en los últimos años se ha visto un crecimiento comercial en negocios de comida, bares, tiendas y licoreras; pero aún no cuentan con un bar que sea un punto de encuentro de amigos, compañeros o conocidos donde no solamente se tome alcohol sino se pueda disfrutar de música en vivo, de la transmisión de eventos deportivos nacionales e internacionales
Se quiere aprovechar el valor de marca que los autores Martín, Bermúdez, Li y Parra (2018), indican que permitirán identificar y a su vez diferenciar la oferta de un bar consolidado en Cuenca; "Safari Licores" respecto a las ofertas existentes en Azogues; el objetivo es brindar un espacio motivado para encuentro de amigos que quieren pasar un momento de tertulia y diversión; dándoles como plus música, video, y la posibilidad de eventos especiales 
con baile y conciertos dentro del local, a eso se refieren los autores Barbopoulos y Johansson (2016), apuntar un objetivo hedónico donde el placer y el estado de júbilo del consumidor le sea más sensible que lo que invierte para eso.

La metodología que se utilizó en esta investigación fue mixta, los autores Ruíz, Borboa y Rodríguez (2013) determinan que las investigaciones mixtas utilizan datos cualitativos y cuantitativos determinándose en 5 fases similares y relacionadas entre sí; llevan a cabo observación y evaluación de fenómenos, establecen suposiciones o ideas como consecuencia de la observación y evaluación realizadas, prueban y demuestran el grado en que las suposiciones $\mathrm{o}$ ideas tienen fundamento, revisan tales suposiciones o ideas sobre la base de las pruebas o del análisis; y por último proponen nuevas observaciones y evaluaciones para establecer, modificar, comentar y/o fundamentar las suposiciones o ideas, o incluso para generar otras

Con datos cualitativos sobre el comportamiento del mercado en Azogues para este tipo de negocios; y cuantitativos para determinar la factibilidad del proyecto.

Los resultados fueron medidos en dos escenarios; el primero con una inversión total por parte de los emprendedores en los cuales se tiene un VAN de US\$ 5,470.75 y un TIR de $20.54 \%$; el segundo escenario es endeudarse en un $50 \%$ con la banca pública o privada y el otro $50 \%$ el aporte de los emprendedores, teniendo como resultado un VAN de US\$ 7,519.72 y una TIR de $28.69 \%$; en ambos escenarios el proyecto tiene factibilidad y es viable.

El emprendimiento es esencial en la actualidad según las autoras Marulanda y Valencia (2016) para la economía de un país; la generación de nuevas empresas se ha convertido en un reto permanente para las diferentes instancias gubernamentales ya que genera nuevos empresarios y abre fuentes de empleo, el fomento de la actividad emprendedora requieres del estudio de motivaciones y percepciones propios del contexto local relacionados con aspectos económicos, culturales y educativos. La investigación actual determina dar dinamismo al mercado de bares en la ciudad de Azogues, activar su vida nocturna y que los habitantes de esta ciudad, las personas que la visiten tengan alternativas de esparcimiento con el presente trabajo se recomienda la ejecución de este proyecto por los resultados obtenidos.

\section{Marco Teórico}

Se define a los bares temáticos o espacios de entretenimiento, bares que brindan diferentes tipos de servicios, no solamente el consumo de alcohol; sino se pueda disfrutar de música en vivo, de la transmisión de eventos deportivos nacionales e internacionales creando innovación para este tipo de negocio. La expresión bares temáticos incluye una serie de opciones que se presentan como novedosas: cibercafés, cafés en museos, casas de arte, disqueras cafés, bares relacionados con algún estilo social.

A esto se agregan lugares ya existentes, que albergan otras actividades habituales de un bar con espectáculos de música, poesía, peñas culturales, algunas actividades nuevas clase de danza, salsa, rumba o tango.

En un aporte de Duarte y Ruíz (2009) consideran que el empleo es uno de los derechos fundamentales que deben dar los gobiernos a sus ciudadanos; para generar condiciones de vida digna y facilitar el desarrollo económico y social del país. Hoy en día los gobiernos promueven los emprendimientos en los distintos niveles de la economía para el desarrollo local y regional en busca de mejorar el bienestar en las sociedades; los sujetos iniciadores de ideas innovadoras causan impacto económico y social posibilitando el crecimiento progresivo no solo en el empleo sino en el nivel productivo, económico y social en las comunidades.

La factibilidad de un proyecto en un aporte del autor Dubs de Moya (2002) considera que el conjunto de actividades vinculadas entre sí, cuya ejecución permitirá el logro de objetivos en atención a las necesidades que pueda tener una institución en un momento determinado, la finalidad del proyecto factible radica en el diseño de una propuesta de acción dirigida a resolver un problema previamente detectada en el entorno.

En un aporte del Valor Actual Neto en una investigación del autor Mete (2014) el VAN es medido como el valor presente de los flujos de efectivo netos de una propuesta, entendiéndose por flujos de efectivo neto la diferencia entre los ingresos y los egresos periódicos. Para actualizar los flujos netos se utiliza una tasa de descuento denominada tasa de expectativa, que es una medida de la rentabilidad mínima exigida por el proyecto que permite recuperar la inversión, cubrir los costos y obtener beneficios.

Un aporte sobre la Tasa Interna de Retorno del autor Altuve (2004) considera a la TIR como el valor relativo que iguala el valor actual de la corriente de ingresos con el valor actual de la corriente de egresos estimados. Es decir, este concepto envuelve criterios de matemáticas financieras al referirse a valores actuales, y criterios contables al mencionar o incluir corrientes de ingresos y egresos.

El objetivo de cuantificar costos y beneficios para los autores Torres y Callegari (2016) es implementar y mejorar la calidad de la información en los proyectos; los resultados permiten describir en forma objetiva el alcance de las metas.

En el estado de arte se han revisado aportes en artículos científicos de autores que han investigado sobre emprendimientos; también se ha revisado los factores importantes para determinar la factibilidad de los proyectos y sus índices de revisión como el VAN, TIR y la razón B/C.

\section{Metodología}

Es una investigación de tipo mixta; utiliza datos cuantitativos y cualitativos para establecer la demanda de este servicio; la metodología desarrollada es la deductiva, va desde el proceso de revisión de todo un mercado a un nicho la recolección de datos de tipo primaria, ya que es extraída directamente de la población del cantón Azogues, 
que están dentro de la edad objetivos del mercado y son clientes potenciales.

La población meta es la del cantón de Azogues, de ambos sexos, que estén entre las edades de 18 a 60 años, estudiantes y profesionales, con y sin empleo.

El marco de muestreo es la totalidad de los habitantes del cantón de Azogues que están comprendida entre las edades de 18 y 60 años.

La técnica de muestreo que se va utilizar en esta investigación el método probabilístico de muestreo aleatorio simple, de poblaciones finitas inferiores a 35.000.

Teniendo los siguientes datos para determinar el tamaño de la muestra, 30.828 habitantes en la ciudad de Azogues de los dos sexos que oscilan entre las edades de 18 a 60 años; el porcentaje que personas que beben licor a nivel nacional es de un $70 \%$ tomado de datos del Instituto Ecuatoriano de Estadísticas y Censos (2010) por lo que sería la probabilidad de éxito de ocurrencia del evento y un $30 \%$ que no bebe el porcentaje de que no ocurra; el margen de confiabilidad que vamos a tener en esta muestra es del $95 \%$.

$$
\mathrm{n}=\frac{Z^{2} \mathrm{PQN}}{e^{2}(N-1)+Z^{2} \mathrm{PQ}}
$$

Teniendo como resultado que la muestra para esta investigación es de 319.

Se realiza una encuesta con las siguientes seis preguntas, aleatoriamente en la ciudad de Azogues

1. ¿Considera que los bares actuales en la ciudad de Azogues brindan una atención?

2. ¿Considera que los precios; determina su decisión de ir a un bar-licorería?

3. ¿Si conociera un bar-licorería en Azogues que le dé una atención preferencial lo visitaría?

4. Considera que además de un bar donde se sirva alcohol también sea un lugar de encuentro

5. ¿Le gustaría que además de poder tomar alcohol, exista música, trasmisión de deportes?

6. ¿Le gustaría que además de compartir con sus amigos exista la posibilidad de tener música en vivo?

Los resultados de la encuesta se determinan en los siguientes figuras:

\section{¿Considera que los bares actuales en la ciudad de Azogues brindan una atención?}

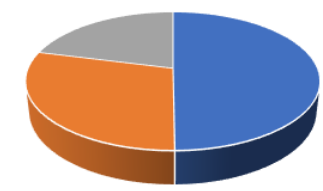

- Buena - Mala - Pésima

Figura 1: Resultados primera pregunta Fuente: Autores

Como se indica en la figura 1 los resultados de la primera pregunta de la encuesta existen un gran porcentaje que considera que la atención de los bares actuales en la ciudad de Azogues es mala y pésima.

\section{¿Considera que los precios determina su decisión de ir a un bar-licorería?}

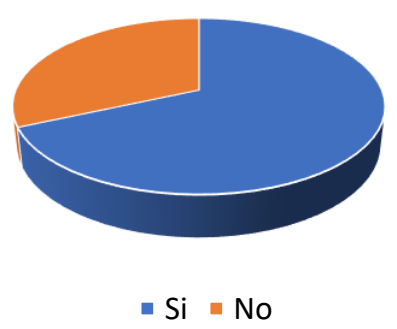

Figura 2: Resultados segunda pregunta Fuente: Autores

Como se indica en la figura 2 los resultados de la segunda pregunta de la encuesta se determinan que el precio si es un factor determinante para tomar la decisión para visitar un bar; relacionando con la primera pregunta al no tener Azogues un bar de características de buena atención, se puede combinar estas dos variables precio/atención para dar un plus a los clientes que buscan este tipo de comercios, especialmente los fines de semana. 


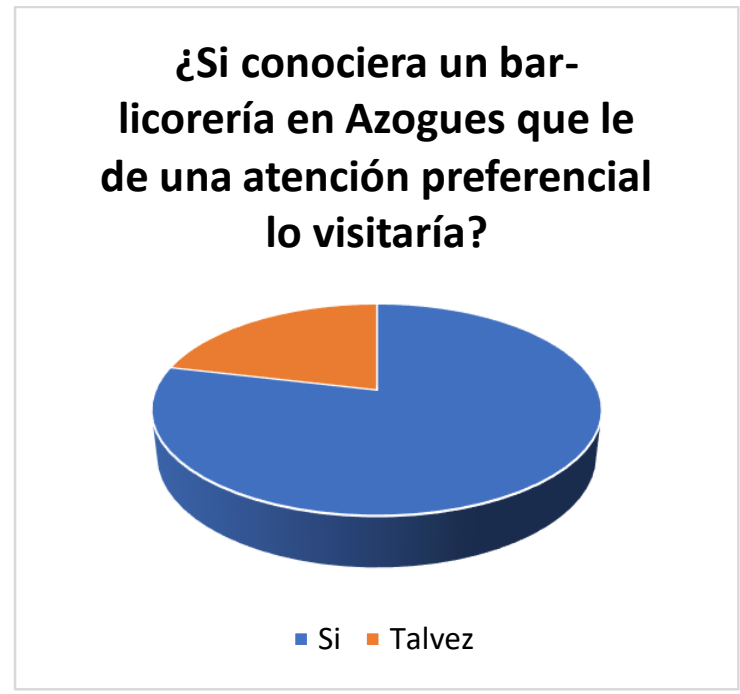

Figura 3: Resultados tercera pregunta

Fuente: Autores

Como se indica en la figura 3 los resultados de la tercera pregunta de la encuesta se constatan que la población del cantón Azogues si visitaría un bar licorería que le ofrezca una atención preferencial y personalizada; el plus de ser atendido con cordialidad y con tiempos mínimos atrae a los clientes para cualquier comercio.

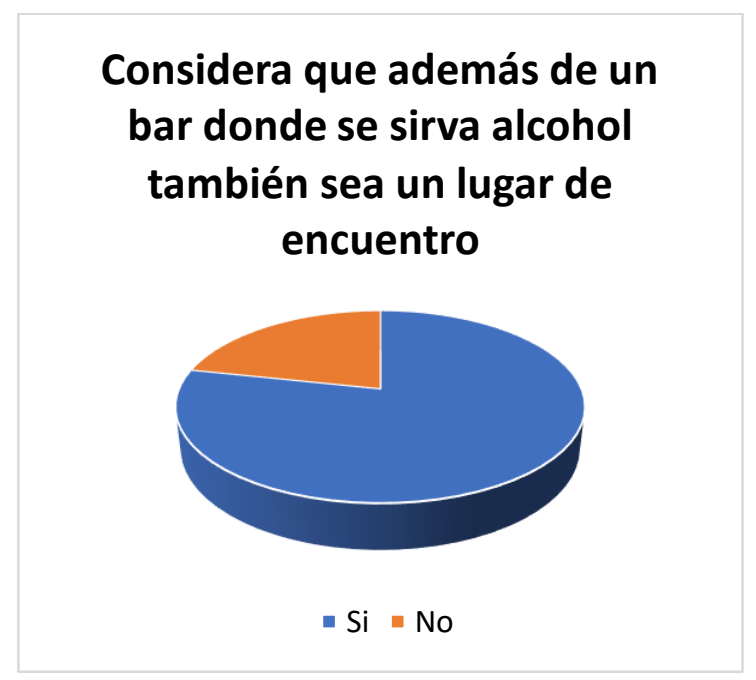

Figura 4: Resultados cuarta pregunta

Fuente: Autores

Como se indica en la figura 4 los resultados de la cuarta pregunta de la encuesta consideran que la población de Azogues busca no solo un lugar donde tomar alcohol, la población necesita un punto de encuentro entre amigos, compañeros de trabajo. y que tenga múltiples alternativas de esparcimiento y diversión.

\section{¿Le gustaría que además de poder tomar alcohol, exista música, trasmisión de deportes?}

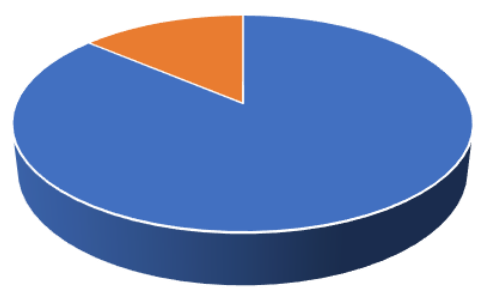

$\because \mathrm{Si} \quad$ No

Figura 5: Resultados quinta pregunta Fuente: Autores

Como se indica en la figura 5 los resultados de la quinta pregunta de la encuesta es que la población de Azogues busca un lugar diferente; que aparte de poder tomar una bebida alcohólica les de alternativas de escuchar música; ver partidos de fútbol o algún otro deporte.

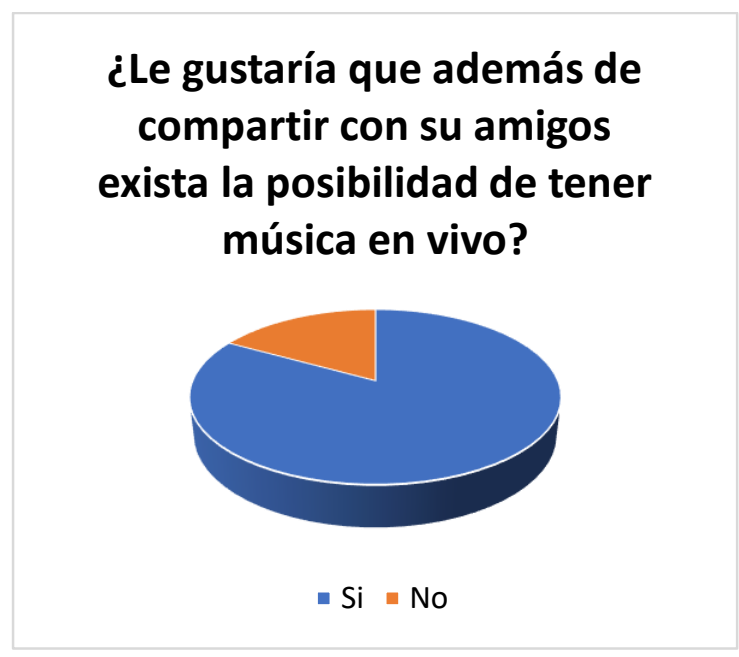

Figura 6: Resultados sexta pregunta Fuente: Autores

Como se indica en la figura 6 los resultados de la sexta pregunta de la encuesta demuestran que la gente en Azogues busca un bar donde tengan la alternativa de escuchar grupos de música en vivo; música de diferentes géneros que amenicen las salidas.

La empresa ya está conformada en la Ciudad de Cuenca y legalizada; se tendría que hacer la apertura de una sucursal debidamente registrada en el SRI y con un adendum notariado de los socios de este nuevo local, deberá tener un representante legal; para la tranquilidad de los inversores. 
En la inversión inicial del arranque de las operaciones en la ciudad de Azogues, se ha revisado los puntos de mayor importancia para dotar al local de un mobiliario acorde a lo que se pretende, dar un espacio nuevo, muy distinto a los locales actuales; los puntos principales para la inversión inicial los siguientes:

- En gastos de operación como arriendos, sueldos, IESS, servicios básicos, internet, se ha previsto una inversión para 6 meses hasta estabilizar el negocio.

- Pagos de permisos de funcionamiento con el Municipio de Azogues y los entes de control correspondientes para este tipo de negocio.

- Activos fijos como, muebles, sillas, TVs, Laptop, frigoríficos, sistema de cámaras de vigilancia, cuadros.

- Se prevé para temas de Marketing Digital y POP, 6 meses de inversión.

- Letreros y cuadros enmarcados en la imagen de "Safari Licores" de Cuenca.

- Intangibles como software de inventarios y facturación.

- Otros como adecuación del local en pintura, y baños.

Con estas explicaciones a continuación el cuadro de la inversión inicial:

Tabla 1: Inversión Inicial

\begin{tabular}{lccc}
\hline Concepto & $\begin{array}{c}\text { Valor } \\
\text { unitario }\end{array}$ & Cantidad & Total \\
\hline Arriendo (6 meses) & $\$ 400,00$ & 6 & $\$ 2.400,00$ \\
Sueldo Facturadora & $\$ 385,00$ & 6 & $\$ 2.310,00$ \\
IESS 12\% Facturadora & $\$ 46,20$ & 6 & $\$ 277,20$ \\
Sueldo Atención al cliente & $\$ 385,00$ & 6 & $\$ 2.310,00$ \\
IESS 12\% Atención al cliente & $\$ 46,20$ & 6 & $\$ 277,20$ \\
Perchas & $\$ 4.000,00$ & 1 & $\$ 4.000,00$ \\
TVs & $\$ 800,00$ & 3 & $\$ 2.400,00$ \\
Laptop Caja; Audio y Video; & $\$ 1.200,00$ & 1 & $\$ 1.200,00$ \\
Sistema cámaras Seguridad & & & \\
Mesas & & & $\$ 2.000,00$ \\
Sillas & & & $\$ 1.000,00$ \\
Frigoríficos Propios & & & $\$ 2.000,00$ \\
Cuadros y adornos & $\$ 50,00$ & 6 & $\$ 300,00$ \\
Implementos Baños & $\$ 500,00$ & 1 & $\$ 500,00$ \\
Letreros & $\$ 200,00$ & 6 & $\$ 1.200,00$ \\
Marketing & & & $\$ 9.800,00$ \\
Inventario Inicial & $\$ 1.000,00$ & 1 & $\$ 1.000,00$ \\
Permisos, patentes, etc & & & $\$ 3.317,44$ \\
Imprevistos 10\% & \multicolumn{1}{c}{ Total de Inversión Inicial } & & $\$ 36.491,84$ \\
\hline \multicolumn{2}{c}{}
\end{tabular}

Fuente: Autores

Para la proyección de ventas se tomó como punto referencial los días de atención de Safari Cuenca; más los datos recolectados del movimiento de la zona en el cual se piensa instalar el negocio en la ciudad de Azogues; por último, aprovechar al máximo las 8 horas laborables del personal en horas de flujo de los potenciales clientes de este tipo de negocios; se plantea la atención en los siguientes horarios:
Tabla 2: Horarios de Atención

\begin{tabular}{cc}
\hline Día & Horario \\
\hline Martes & $14: 00-22: 00$ \\
Miércoles & $14: 00-22: 00$ \\
Jueves & $14: 00-24: 00$ \\
Viernes & $14: 00-2: 00$ \\
Sábado & $14: 00-2: 00$ \\
\hline
\end{tabular}

Fuente: Autores

Con un histórico de ventas en "Safari Cuenca"; se determina que por cliente existe un consumo promedio de US $\$ 5$; pronosticando que en días de baja afluencia; martes y miércoles se podría alcanzar un promedio de 60 clientes; los jueves subir a 100; y los fines de semana viernes y sábado llegar a 200.

No se considera que entre semana se puedan desarrollar eventos deportivos que concentren al público, no se contempla los feriados nacionales y locales que aumentan la demanda, pero tampoco se estima los días no se permite la apertura por ley seca en caso de elecciones; sin tomar en cuenta las variables mencionadas, se prevé las siguientes ventas:

Tabla 3: Pronóstico de las ventas

\begin{tabular}{ccc}
\hline Día & Horario & Ventas \\
\hline Martes & $14: 00-22: 00$ & $\$ 300,00$ \\
Miércoles & $14: 00-22: 00$ & $\$ 300,00$ \\
Jueves & $14: 00-24: 00$ & $\$ 500,00$ \\
Viernes & $14: 00-2: 00$ & $\$ 1.000,00$ \\
Sábado & $14: 00-2: 00$ & $\$ 1.000,00$ \\
\hline & Semanal & $\$ 3.100,00$ \\
& Semanas & 4 \\
\hline
\end{tabular}

Fuente: Autores

Se obtuvo el dato de que en este tipo de negocio existe un margen de ganancia del $30 \%$ se tendría un presupuesto de ventas, otro de costo de ventas y un último de margen de utilidad de la siguiente manera:

Tabla 4: Presupuesto de las ventas, costo y utilidad bruta mensuales

\begin{tabular}{ccccc}
\hline Día & Horario & Ventas & $\begin{array}{c}\text { Rentabilidad } \\
30 \%\end{array}$ & $\begin{array}{c}\text { Costo de } \\
\text { Ventas }\end{array}$ \\
\hline Martes & $14: 00-22: 00$ & $\$ 300,00$ & $\$ 90,00$ & $\$ 210,00$ \\
Miércoles & $14: 00-22: 00$ & $\$ 300,00$ & $\$ 90,00$ & $\$ 210,00$ \\
Jueves & $14: 00-24: 00$ & $\$ 500,00$ & $\$ 150,00$ & $\$ 350,00$ \\
Viernes & $14: 00-2: 00$ & $\$ 1.000,00$ & $\$ 300,00$ & $\$ 700,00$ \\
Sábado & $14: 00-2: 00$ & $\$ 1.000,00$ & $\$ 300,00$ & $\$ 700,00$ \\
\hline & Semanal & $\$ 3.100,00$ & $\$ 930,00$ & $\$ 2.170,00$ \\
& Semanas & 4 & 4 & 4 \\
& Mensual & $\$ 12.400,00$ & $\$ 3.720,00$ & $\$ 8.680,00$ \\
\hline
\end{tabular}

Fuente: Autores

Los gastos operacionales, contando con 2 empleados, tomando el pago de IESS, impuesto al SRI; los décimos y 
un cálculo aproximado de horas extras de los días jueves, viernes y sábado.

Tabla 5: Gastos Operacionales mensuales

\begin{tabular}{lccc}
\hline & Básico & Empleados & Año 1 \\
\hline${ }^{*}$ Gastos Sueldos & $\$ 394,00$ & 2 & $\$ 788,00$ \\
Agua & $\$ 20,00$ & & $\$ 20,00$ \\
Internet & $\$ 50,00$ & & $\$ 50,00$ \\
Cable & $\$ 40,00$ & & $\$ 40,00$ \\
Luz & $\$ 80,00$ & & $\$ 80,00$ \\
Celular & $\$ 40,00$ & & $\$ 40,00$ \\
$*$ Arriendo & $\$ 400,00$ & & $\$ 400,00$ \\
$*$ Implementos Baños & $\$ 50,00$ & & $\$ 50,00$ \\
$*$ Marketing & $\$ 200,00$ & & $\$ 200,00$ \\
$*$ Pago IVA & $\$ 446,40$ & & $\$ 446,40$ \\
$*$ Pagos IESS & $\$ 58,57$ & 2 & $\$ 117,15$ \\
$*$ Horas Extras & $\$ 40,00$ & 2 & $\$ 262,67$ \\
$*$ Decimo XIII & $\$ 43,78$ & 2 & $\$ 87,56$ \\
$*$ Decimo XIV & $\$ 394,00$ & 2 & $\$ 65,67$ \\
$*$ Fondos de Reserva & $\$ 0,00$ & 2 & $\$ 0,00$ \\
$*$ Vacaciones & $\$ 21,89$ & 2 & $\$ 43,78$ \\
\hline \multicolumn{2}{l}{ Gasto Total Mes } & & $\$ 2.691,22$ \\
\hline
\end{tabular}

Los presupuestos de ventas, costo de ventas; y gastos operacionales a continuación un presupuesto anual de todos estos datos y un aproximado de la utilidad neta, recordando que esta utilidad neta esta antes de pago de impuestos a la renta y participación de empleados.

Tabla 6: Presupuesto de las ventas, costo y utilidad operacional anuales

\begin{tabular}{cccccc}
\hline Mes & Ventas & $\begin{array}{c}\text { Costo de } \\
\text { Ventas }\end{array}$ & U. Bruta & Gastos & $\begin{array}{c}\text { Utilidad } \\
\text { Neta }\end{array}$ \\
\hline 1 & $\$ 12.400,00$ & $\$ 8.680,00$ & $\$ 3.720,00$ & $\$ 2.691,22$ & $\$ 1.028,78$ \\
2 & $\$ 12.400,00$ & $\$ 8.680,00$ & $\$ 3.720,00$ & $\$ 2.691,22$ & $\$ 1.028,78$ \\
3 & $\$ 12.400,00$ & $\$ 8.680,00$ & $\$ 3.720,00$ & $\$ 2.691,22$ & $\$ 1.028,78$ \\
4 & $\$ 12.400,00$ & $\$ 8.680,00$ & $\$ 3.720,00$ & $\$ 2.691,22$ & $\$ 1.028,78$ \\
5 & $\$ 12.400,00$ & $\$ 8.680,00$ & $\$ 3.720,00$ & $\$ 2.691,22$ & $\$ 1.028,78$ \\
6 & $\$ 12.400,00$ & $\$ 8.680,00$ & $\$ 3.720,00$ & $\$ 2.691,22$ & $\$ 1.028,78$ \\
7 & $\$ 12.400,00$ & $\$ 8.680,00$ & $\$ 3.720,00$ & $\$ 2.691,22$ & $\$ 1.028,78$ \\
8 & $\$ 12.400,00$ & $\$ 8.680,00$ & $\$ 3.720,00$ & $\$ 2.691,22$ & $\$ 1.028,78$ \\
9 & $\$ 12.400,00$ & $\$ 8.680,00$ & $\$ 3.720,00$ & $\$ 2.691,22$ & $\$ 1.028,78$ \\
10 & $\$ 12.400,00$ & $\$ 8.680,00$ & $\$ 3.720,00$ & $\$ 2.691,22$ & $\$ 1.028,78$ \\
11 & $\$ 12.400,00$ & $\$ 8.680,00$ & $\$ 3.720,00$ & $\$ 2.691,22$ & $\$ 1.028,78$ \\
12 & $\$ 12.400,00$ & $\$ 8.680,00$ & $\$ 3.720,00$ & $\$ 2.691,22$ & $\$ 1.028,78$ \\
\hline Totales & $\$ 148.800,00$ & $\$ 104.160,00$ & $\$ 44.640,00$ & $\$ 32.294,59$ & $\$ 12.345,41$ \\
\hline
\end{tabular}

La factibilidad del proyecto se debe medir al mediano plazo es decir a 5 años, para analizar el VAN y el TIR y recomendar si el proyecto es viable o rechazarlo.

Con los siguientes datos obtenidos hasta el momento, más datos del comercio y de la banca actual, se realiza la revisión de la factibilidad del proyecto en dos escenarios:

1) Con la inversión neta del dinero por parte de los inversionistas.

2) Con la inversión del $50 \%$ de los inversionistas y un $50 \%$ con préstamos de alguna entidad bancaria.
Con la inversión neta del dinero por parte de los inversionistas.

- Inversión inicial: US\$36.491,84

- Porcentaje Residual de Depreciación: 10

- Vida Fiscal: 5 años.

- Impuesto a la Renta: $22 \%$

- Utilidad primer año: US\$ 12.345.41

- Incremento de ventas anual: $10 \%$

- Tasa relativa de Interés de consumo sector bancaria: 15 Con la fórmula del VAN:

$$
\mathrm{VAN}=\frac{D}{(1+i)}+\frac{D}{(1+i)^{2}}+\cdots+\frac{D_{n}}{(1+i)^{n}}
$$

La fórmula de la TIR:

$$
\mathrm{TIR}=\sum_{T=0}^{n} \frac{F_{n}}{(1+i)^{n}}=0
$$

Y la de la razón Beneficio/Costo:

$$
\mathrm{B} / \mathrm{C}=\frac{B-B N-M \& O}{I-V S}
$$

Tabla 7: Cálculos del VAN, TIR y B/C con inversión total de los socios

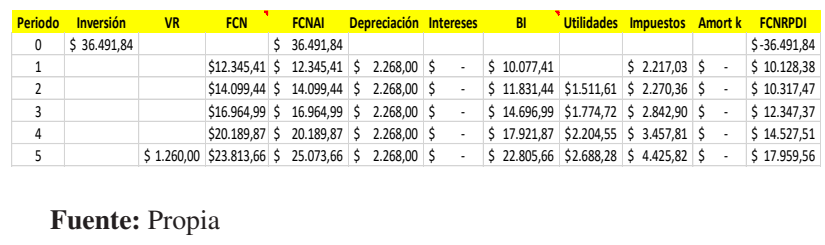

Teniendo como resultado que el VAN (Valor Actual Neto) es positivo en US\$ 5470.75; la TIR (Tasa Interna de Retorno) es del $20.54 \%$ mayor a la tasa nominal de interés de consumo bancaria y la razón $\mathrm{B} / \mathrm{C}$ es 1.37 mayor a 1 ; el proyecto es viable y rentable.

Con la inversión del $50 \%$ de los inversionistas y un $50 \%$ con préstamos de alguna entidad bancaria.

Con los siguientes datos se desarrolla el proyecto de factibilidad:

- Inversión inicial 50\% inversionistas US\$18.245,92

- Inversión inicial $50 \%$ préstamo bancario US\$18.245,92

- Porcentaje Residual de Depreciación: $10 \%$

- Vida Fiscal: 5 años.

- Impuesto a la Renta: $22 \%$

- Utilidad primer año: US\$12.345.41

- Incremento de ventas anual: $10 \%$

- Tasa relativa de Interés de consumo sector bancaria: $15 \%$ 
Tabla 8: Cálculo de los intereses del préstamo del $50 \%$ de la inversión

\begin{tabular}{rrrrrr}
\hline $\mathbf{N}$ & K inicial & Amort k & Intereses & Pago & Saldo k \\
\hline $\mathbf{1}$ & $\$ 18.245,92$ & $\$ 2.706,15$ & $\$ 2.736,89$ & $\$ 5.443,04$ & $\$ 15.539,77$ \\
$\mathbf{2}$ & $\$ 15.539,77$ & $\$ 3.112,08$ & $\$ 2.330,96$ & $\$ 5.443,04$ & $\$ 12.427,69$ \\
$\mathbf{3}$ & $\$ 12.427,69$ & $\$ 3.578,89$ & $\$ 1.864,15$ & $\$ 5.443,04$ & $\$ 8.848,80$ \\
$\mathbf{4}$ & $\$ 8.848,80$ & $\$ 4.115,72$ & $\$ 1.327,32$ & $\$ 5.443,04$ & $\$ 4.733,08$ \\
$\mathbf{5}$ & $\$ 4.733,08$ & $\$ 4.733,08$ & $\$ 709,96$ & $\$ 5.443,04$ & $\$$ \\
\hline
\end{tabular}

Fuente: Propia

La tabla 8 refleja la amortización del pago del capital y de los intereses del préstamo bancario que podrían adquirir los socios para el emprendimiento del negocio; para 5 años.

Tabla 9: Cálculos del VAN, TIR y B/C con la inversión del $50 \%$ de los socios

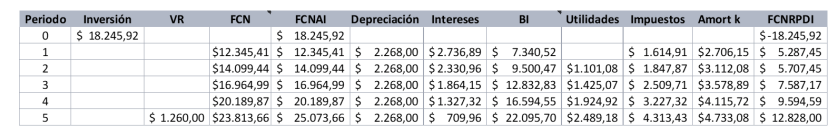

Fuente: Propia

Teniendo como resultado que el VAN (Valor Actual Neto) es positivo en US\$ 7519.72; la TIR (Tasa Interna de Retorno) es del $28.69 \%$ mayor a la tasa nominal de interés de consumo bancaria y la razón $\mathrm{B} / \mathrm{C}$ es 1,91 mayor a 1 ; el proyecto es viable y rentable.

\section{Resultados}

Los resultados de la investigación del emprendimiento son:

- Con la inversión neta del dinero por parte de los inversionistas.
○ VAN: US\$ 5470.75
○ TIR: $20.54 \%$
○ B/C: 1.37

- Con la inversión del $50 \%$ de los inversionistas y un $50 \%$ con préstamos de alguna entidad bancaria.
○ VAN: US\$7519.72
- TIR: $28.69 \%$
○ B/C: 1.91

\section{Conclusiones y Recomendaciones}

El proyecto es novedoso por las condiciones de distinción del bar que se desea instalar; a diferencia a los bares actuales en la ciudad de Azogues; el sector donde se pretende colocar el negocio es de un alto crecimiento económico en la ciudad. Por los resultados positivos en los dos escenarios expuestos en la investigación, del VAN, TIR y la razón $\mathrm{B} / \mathrm{C}$ el proyecto es rentable al mediano plazo 5 años.

La inversión inicial es alta por que se toma 6 meses de sueldos de las 2 personas que se necesita para atender el bar; publicidad de igual manera para 6 meses hasta tomar posicionamiento del negocio.

Se recomienda ejecutar el Proyecto con una combinación de capital propio y financiamiento de la banca pública como BanEcuador por tasas más convenientes; emplazar el local en la zona de expansión de la ciudad de Azogues.

\section{Referencias Bibliográficas}

Altuve, J. (2004). El uso del valor actual neto y la tasa interna de retorno para la valoración de las decisiones de inversión. Actualidad Contable Faces, 7(9), 7-17. Descargado de http://www.redalyc .org/articulo.oa?id=25700902

Barbopoulos, I., y Johansson, L. (2016). A multidimensional approach to consumer motivation: exploring economic, hedonic, and normative consumption goals. Journal of Consumer Marketing, 33, 75-84. doi: http://dx.doi.org/10.1108/JCM-08-2014 $-1091$

de Estadísticas y Censos, I. E. (2010). Datos demográficos del ecuador.

Duarte, T., y Ruiz, M. (2009). Emprendimiento, una opción para el desarrollo. Scientia Et Technica, 15(43), 3262331. Descargado de http://www.redalyc . org/articulo.oa?id=84917310058

Dubs de Moya, R. (2002). El proyecto factible: una modalidad de la investigación. Revista Universitaria de Investigación, 3, 2. Descargado de http: / / www.redalyc.org/articulo . oa? id=41030203

Martín, M., Bermúdez, G., Li, Z., y Parra, F. (2018). Modelo de administración de marcas: un enfoque teórico basado en la teoría general de sistemas. Esic Market Economics and Business Journal, 49, 1. Descargado de https://www.researchgate . net/profile/Manuel_Martin11/ publication/32389836

Marulanda, F., y Morales, S. (2016). Entorno y motivaciones para emprender. Revista Escuela de Administración de Negocios Universidad EAN, 81, 12-28. Descargado de http://www.redalyc . org/articulo.oa?id=20649705002

Mete, M. (2014). Valor actual neto y tasa de retorno: $\mathrm{Su}$ utilidad como herramientas para el análisis y evaluación de proyectos de inversión. Fides el Ratio, 7, 67-85. Descargado de http:// www.scielo.org.bo/scielo.php?pid= s2071-081X2014000100006\&script=sci -arttext

Ruíz, M., Borboa, M., y Rodríguez, J. (2013). El enfoque mixto de investigación en los estudios fiscales. Revista Académica de Investigación, 13, 1-25. Descargado de http:// www. eumed.net/rev/tlatemoani/13/ estudios-fiscales.pdf 
Torres, C., y Callegari, N. (2016). Criterios para cuantificar costos y beneficios en proyectos de mejora de calidad. Instituto Superior Técnico José Antonio Echeverría, 37(2), 151-163. Descargado de http: / / www.redalyc.org/articulo .oa?id=360446197005

Recibido: 6 de septiembre de 2020

Aceptado: 13 de octubre de 2020

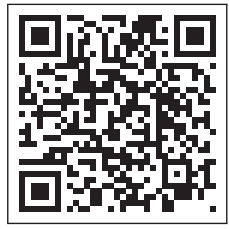

